\title{
Universiteit
}

Leiden

The Netherlands

\section{Dynamic response of trapped ultracold bosons on optical lattices}

Batrouni, G.G.; Assaad, F.F.; Scalettar, R.T.; Denteneer, P.J.H.

\section{Citation}

Batrouni, G. G., Assaad, F. F., Scalettar, R. T., \& Denteneer, P. J. H. (2005). Dynamic response of trapped ultracold bosons on optical lattices. Physical Review A, 72(3), 031601. doi:10.1103/PhysRevA.72.031601

Version: $\quad$ Not Applicable (or Unknown)

License: $\quad$ Leiden University Non-exclusive license

Downloaded from: https://hdl.handle.net/1887/61307

Note: To cite this publication please use the final published version (if applicable). 


\title{
Dynamic response of trapped ultracold bosons on optical lattices
}

\author{
G. G. Batrouni, ${ }^{1}$ F. F. Assaad, ${ }^{2}$ R. T. Scalettar, ${ }^{3}$ and P. J. H. Denteneer ${ }^{4}$ \\ ${ }^{1}$ Institut Non-Linéaire de Nice, Université de Nice-Sophia Antipolis, 1361 Route des Lucioles, 06560 Valbonne, France \\ ${ }^{2}$ Institut für Theoretische Physik und Astrophysik, Universität Würzburg, Am Hubland, D-97074 Würzburg, Germany \\ ${ }^{3}$ Physics Department, University of California, Davis, California 95616, USA \\ ${ }^{4}$ Lorentz Institute, Leiden University, P. O. Box 9506, 2300 RA Leiden, The Netherlands
}

(Received 16 March 2005; published 14 September 2005)

\begin{abstract}
We study the dynamic response of ultracold bosons trapped in one-dimensional optical lattices using Quantum Monte Carlo simulations of the boson Hubbard model with a confining potential. The dynamic structure factor reveals the inhomogeneous nature of the low temperature state, which contains coexisting Mott insulator and superfluid regions. We present new evidence for local quantum criticality and discuss implications for the experimental excitation spectrum of ${ }^{87} \mathrm{Rb}$ atoms confined in one dimension.
\end{abstract}

DOI: 10.1103/PhysRevA.72.031601

PACS number(s): 03.75.Kk, 03.75.Lm, 03.75.Hh, 05.30.Jp

The advent of Bose-Einstein condensation in cold atomic gases opened new fields of research merging atomic physics and quantum optics with condensed matter physics. Scattering experiments common in solid state physics and liquid helium have been adapted to extract information on excitation dynamics of atomic condensates resulting in the technique of Bragg spectroscopy[1]. Initial experiments on Bosecondensed gases could be well described by mean-field Gross-Pitaevskii approaches, valid for weak interactions. However, recent experiments have reached the regime of strongly interacting atoms, often by confining the atoms in optical lattices, leading, for instance, to the destruction of a phase-coherent superfluid (SF) phase and the establishment of Mott insulator (MI) phases with squeezed number fluctuations [2-4]. The latter state is considered as promising for use as a large quantum register for quantum information purposes [5].

Ultracold atoms in optical lattices are an almost ideal realization of the boson Hubbard model [6], that has previously been studied intensely (with different motivation) $[7,8]$. Much of this previous work focussed on the equilibrium phase diagram or on the conductivity in the presence of disorder. However, as in other condensed matter systems, a rich source of information on the excitation dynamics is the dynamic structure factor, the double Fourier transform of density-density correlations, which can be measured using Bragg spectroscopy $[1,9,10]$. It is therefore of great current interest to study the dynamic structure factor of the boson Hubbard model. Because of loss of translational invariance due to the presence of the confining trap, results for the uniform system cannot be directly applied to the confined system. This affects both the static response, the system no longer has a globally incompressible phase [11], and the dynamics, since the wave vector is no longer a good quantum number.

In this paper, we use Quantum Monte Carlo to study the ground state of the one-dimensional boson-Hubbard model in a confining harmonic potential,

$$
\begin{aligned}
H= & -t \sum_{i}\left(a_{i}^{\dagger} a_{i+1}+a_{i+1}^{\dagger} a_{i}\right)+V_{T} \sum_{i}\left(x_{i}-\frac{L}{2}\right)^{2} n_{i} \\
& +U \sum_{i} n_{i}\left(n_{i}-1\right),
\end{aligned}
$$

where $L$ is the number of sites and $x_{i}$ is the coordinate of the $i$ th site. The hopping parameter, $t$, sets the energy scale, $n_{i}$ $=a_{i}^{\dagger} a_{i}$ is the number operator, $\left[a_{i}, a_{j}^{\dagger}\right]=\delta_{i j}$ are bosonic creation and destruction operators. $V_{T}$ sets the confining trap curvature, while the contact interaction is given by $U$. We use the World Line algorithm for our simulations. The static properties and state diagram of this model were studied in [11] where it was shown, among other things, that the presence of the trap and the destruction of translation invariance
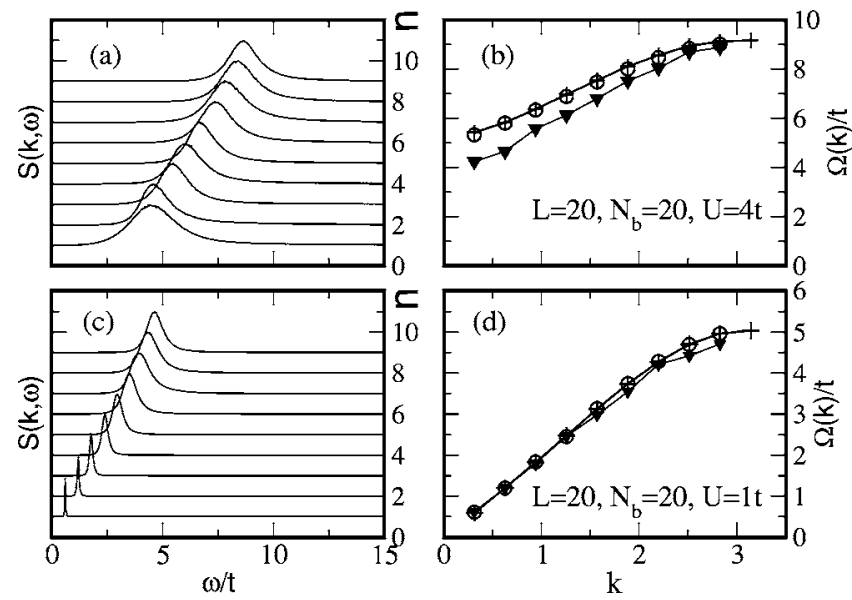

FIG. 1. (a,c) The dynamic structure factor $S(k, \omega)$ vs $\omega$ for $k=2 \pi n / L$ with $1 \leqslant n \leqslant 9$. For better visibility, the curves are shifted for the different values of $n$ shown on the right vertical axes. (b,d) the dispersion $\Omega(k)$ vs $k$ for the same values of $k$. The circles are obtained with Eq. (3), the pluses with Eqs. (5) and (6). The down triangles are the positions of the maxima of $S(k, \omega)$ in $(\mathrm{a}, \mathrm{c})$. The system has commensurate filling: $L=20, N_{b}=20, \beta=10$ with $U$ $=4 t(\mathrm{a}, \mathrm{b})$ and $U=1 t(\mathrm{c}, \mathrm{d})$. 


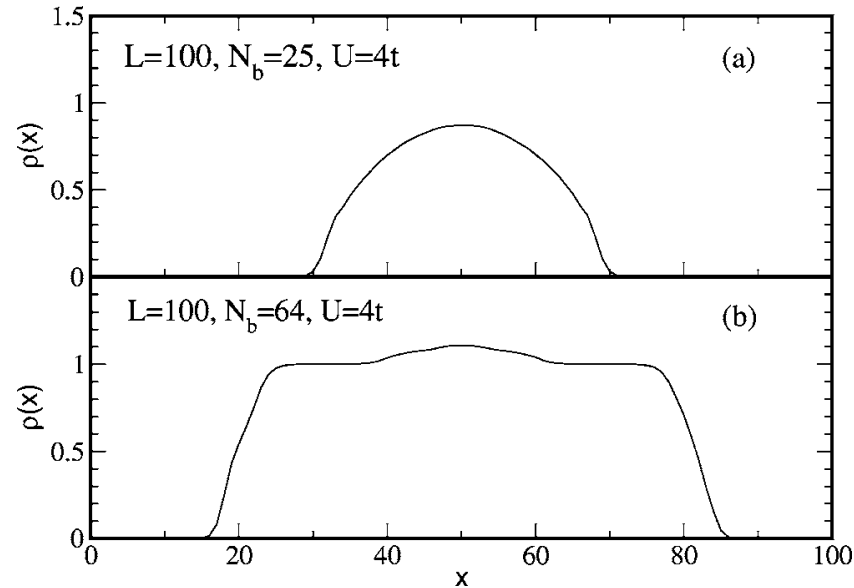

FIG. 2. Local density profile, $\rho(x)$, for the confined system with $L=100, V_{T}=0.008, U=4 t$ : (a) $N_{b}=25$ (pure SF) and (b) $N_{b}=64$ (coexistence SF-MI).

eliminate the quantum phase transitions between the SF and MI phases and replace it by a coexistence of phases. Our main results are: (i) due to coexistence of MI and SF regions in the confined system, the dynamic structure factor exhibits two excitation branches, one gapped and one with a linear, phononlike, dispersion; (ii) these branches are shown clearly to be connected to the spatial regions containing the MI and SF phases; and (iii) by using an appropriate measure of local excitations, we provide new evidence for local quantum criticality.

In the ground state $(T=0)$ the dynamic structure factor is given by

$$
\begin{aligned}
S(k, \omega) & =\frac{1}{L^{2} L_{t}} \sum_{t} \sum_{r, r^{\prime}} \mathrm{e}^{-i \omega t} \mathrm{e}^{i k\left(r-r^{\prime}\right)}\left\langle n(r, 0) n\left(r^{\prime}, t\right)\right\rangle \\
& =\sum_{m} \delta\left(\omega-E_{m}+E_{0}\right)|\langle 0|n(k, 0)| m\rangle|^{2},
\end{aligned}
$$

where $L_{t}$ is the number of time slices, $E_{m}$ the energy of state
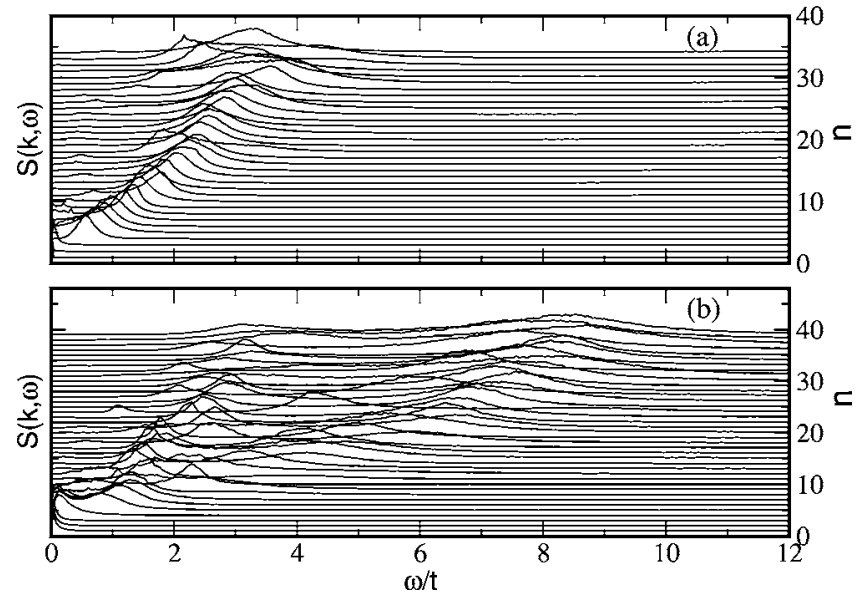

FIG. 3. $S(k, \omega)$ vs $\omega(k=2 \pi n / L)$ for the system in Fig. 2, $N_{b}=25$ (a) and $N_{b}=64$ (b). The two ridges in (b) reflect the SF-MI co-existence, Fig. 2(b). Same representation is used as in Fig. 1.

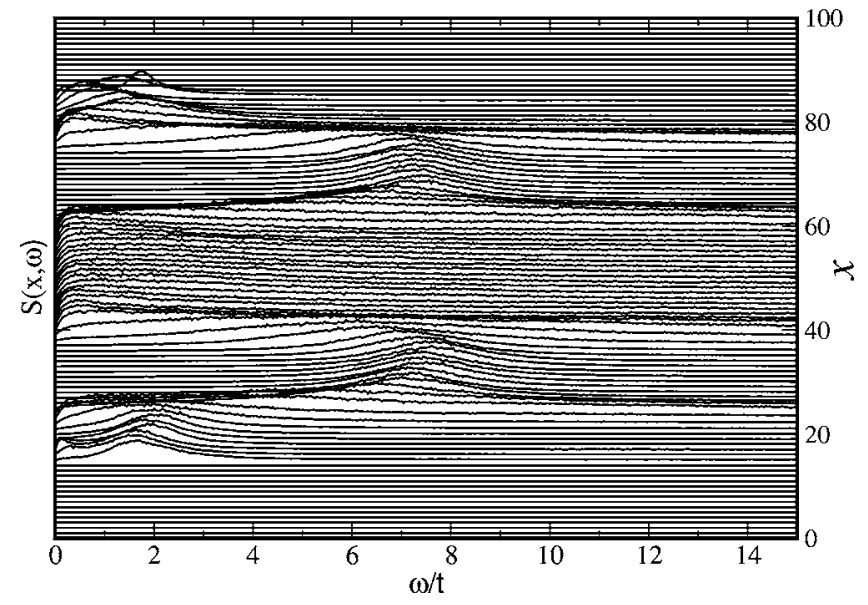

FIG. 4. $S(x, \omega)$ for the system in Fig. 3(b). The origin of gapped and ungapped excitation branches are clearly identified with the SF and MI regions. The curves are shifted for the different values of $x$ which are shown on the right vertical axis.

$|m\rangle$, and $\left\langle n(r, 0) n\left(r^{\prime}, t\right)\right\rangle$ the space and time separated density-density correlation function. With our normalization we have $\int \mathrm{d} \omega S(k, \omega)=N_{b} S(k)$ where $S(k)$ is the static structure factor and $N_{b}$ the number of bosons. Equation (2) shows that, for a given $k$, the peaks in $S(k, \omega)$ correspond to the excitation energies. We have performed all our simulations at $\beta=T^{-1}=10$ and verified that it is sufficient to study the ground state. In the World Line algorithm, it is a simple matter to measure $\left\langle n(r, 0) n\left(r^{\prime}, \tau\right)\right\rangle$ where $\tau$ is the imaginary time separation. The dynamic structure factor is then given by Eq. (2), with $t$ replaced by $-i \tau$ which then requires the performance of a Laplace, instead of a Fourier, transform. This is done with the help of the stochastic analytic continuation method [12].

Figure 1 shows $S(k, \omega)$ for the uniform system in the MI (a) and SF (c) phases. The critical value in one dimension is $U_{\text {crit }}=2.33 t[13]$. Note that, for better visibility, we have nor-

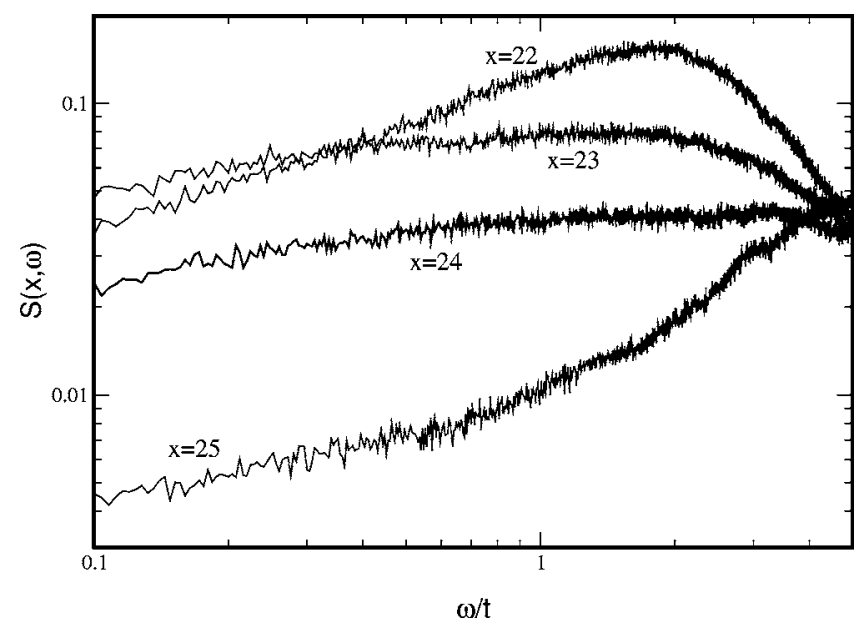

FIG. 5. $S(x, \omega)$ for the system in Fig. 3(b) at the four indicated sites where one of the transitions from SF to MI is observed. For both $x=23,24, S(x, \omega)$ is constant for more than a decade in $\omega$ which means that its Laplace transform decays as $\tau^{-1}$. 
malized the structure factor curves in Figs. 1, 3, and 4 such that the maximal peak height is unity. Consequently the structure factor curves we show are dimensionless and do not show the true spectral weights. For the SF, it is clear that the excitation energies for small $k$ are linear in $k$, i.e., phononic, leading to a stable SF phase (according to the Landau criterion). On the other hand, the MI case clearly shows a gap at $\omega \approx 3 t$, where the spectral weight in Fig. 1(a) starts to be appreciable. This is in excellent agreement with the value of the gap of $3 t$ measured in [8] from the static compressibility. Note that the first peak is at $\omega \approx 4.5 t$, which is larger than the gap, and that the peaks for the MI are rather wide. This is due to the presence of several closely spaced peaks which maximum entropy has difficulty resolving. Such closely spaced peaks are seen in the exact diagonalization of smaller systems [10] where, for the MI, the first in the lowest group of peaks does indeed correspond to the gap.

One can study the excitation spectrum using the dispersion relation $\Omega(k)$,

$$
\Omega(k)=\frac{\int \mathrm{d} \omega \omega S(k, \omega)}{\int \mathrm{d} \omega S(k, \omega)},
$$

which can be expressed in terms of purely static quantities using the $f$-sum rule

$$
\begin{gathered}
\int_{-\infty}^{+\infty} \mathrm{d} \omega \omega S(k, \omega)=N_{b} E_{k}, \\
E_{k}=\frac{-t}{L}(\cos (2 \pi k / L)-1)\left\langle 0\left|\sum_{i=1}^{L}\left(a_{i}^{\dagger} a_{i+1}+a_{i+1}^{\dagger} a_{i}\right)\right| 0\right\rangle .
\end{gathered}
$$

The dispersion relation is then given by the Feynman result,

$$
\Omega(k)=\frac{E_{k}}{S(k)} .
$$

Figure 1 shows $\Omega(k)$ obtained using the dynamic structure factor, Eq. (3) (circles), and also using only static quantities, Eqs. (5) and (6) (pluses) in the MI (b) and SF (d) phases. These two calculations agree very well and give for the SF phase linear dispersion for small $k$ and a gap for the MI. While $\Omega(k)$ shows clearly the presence of a gap for MI, it does not give its value since it is an integral over all peaks that may be present. In addition, we show the positions of the peaks in both cases (down triangles) which agree extremely well with $\Omega(k)$ when the peaks are very sharp. This offers a consistency and precision check on our methods. Our results for $\Omega(k)$ agree with the small lattice exact diagonalization results [10].

We now turn to the confined system where we always take a system size $L=100$ and $V_{T}=0.008$. These values and the fillings we use are comparable to the experimental ones. Figure 2 shows the local density profiles for a system in a pure SF phase, (a), and with coexistence of SF and MI, (b). Figure 3 shows the corresponding $S(k, \omega)$. We note that although $k$ is not a good quantum number due to loss of trans- lational invariance, computing $S(k, \omega)$ as in Eq. (2) remains meaningful even with a trap $[4,14]$. For the pure SF case, Fig. 3(a), $S(k, \omega)$ is similar to the SF case in Fig. 1(c): no gap is visible and the excitation energies go to zero smoothly as $k \rightarrow 0$. Figure 3(b) shows the same for $N_{b}=64$ where there is coexistence of MI and SF phases. There is a marked difference in the behavior of $S(k, \omega)$ compared to the pure SF case: two branches of excitation peaks are clearly visible in the form of two ridges. The low energy branch is seen to be very similar to $S(k, \omega)$ in the SF case, Fig. 3(a), saturating at about $\omega \approx 3 t$, and going smoothly to zero. The second family of peaks [15] saturates at a higher energy, $\omega \approx 8 t$ and seems to make its first appearance at about $k \approx 11$ in units of $2 \pi / L$ with an $\omega \approx 3 t$.

To interpret these two excitation branches, we examine $S(x, \omega)$, the imaginary time Laplace transform of $\langle n(x, 0) n(x, \tau)\rangle$, the same site but time-separated densitydensity correlation function. This is shown in Fig. 4 which clearly identifies excitation branches with position in the trap. $S(x, \omega)$ includes contributions from all $k$ values. The very low energy phonon excitations arise from the SF regions in the outer wings and the center of the system. The middle excitations, $\omega \sim 2 t-3 t$ appear prominently in the squeezed SF region between the MI and the outer edges of the system. The value of $\omega$ at the peak corresponds well to the saturation value, at high $k$, of the SF branch of $S(k, \omega)$ in Fig. 3. This effect was observed in all our simulations where a MI phase is present. In these outer regions the superfluid is squeezed between the MI and the rapidly increasing confining potential which appears to enhance the spectral weight of the high $\omega$ excitations and make them so prominent. Finally, the peaks in the two MI regions in Fig. 4 agree well with the saturation value of the higher $\omega$ branch in Fig. 3(b). This confirms our interpretation that this branch corresponds to excitations in the Mott phase. As further evidence, we mention that the $S(x, \omega)$ for the uniform system in the MI phase at $U=4 t$ [Fig. 1(a)] has its peak at $\omega \approx 8 t$, like the Mott regions in Fig. 3(b).

The interpretation of the higher $\omega$ excitation branch in $S(k, \omega)$ as being due to the MI regions (Fig. 4) and the absence of a gap in Fig. 3 show that even when MI regions are present, the system is gapless! Figure 4 shows that local excitation energies in the MI regions correspond to those in MI phase of the uniform system at the same $U$.

Another very striking feature in Fig. 4 is the behavior of $S(x, \omega)$ which appears to be very broad at the boundary between SF and MI sites. To exhibit this in more detail, we show $S(x, \omega)$ in Fig. 5 for four sites where a transition from SF to MI is observed. It is clear that the behavior of $S(x, \omega)$ is very different at sites $x=23$ and 24 where it is almost constant for about a decade in $\omega$. This means that the Laplace transform of $S(x, \omega)$ at these sites behaves as a power law, $S(x, \tau) \sim \tau^{-1}$, indicating diverging correlations in the imaginary time direction and thus local criticality. The big contrast with the form of $S(x, \omega)$ at the neighboring sites (22 and 25) shows that the transition from SF to MI takes place over only a few sites (two in this case). This is renewed evidence of the local quantum criticality already discussed in $[11,16]$. 


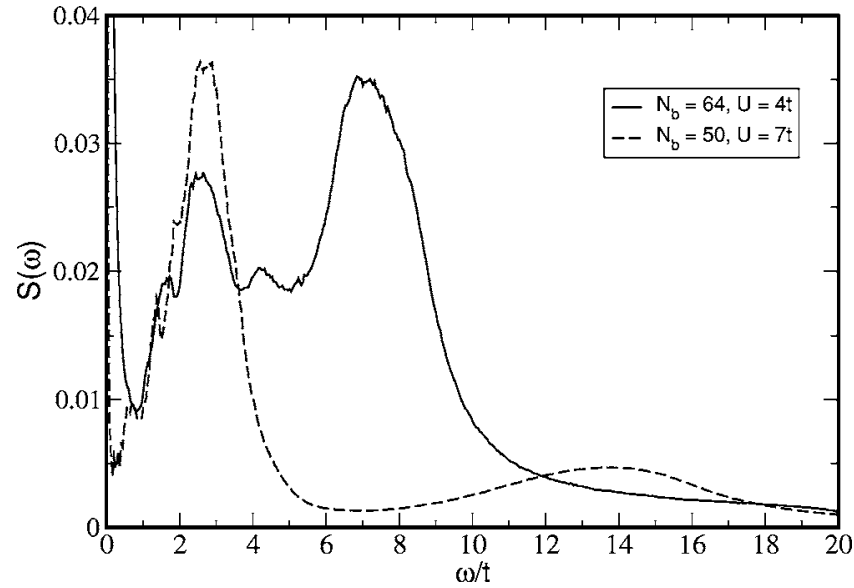

FIG. 6. $S(\omega)$ for $N_{b}=64, U=4 t$ and for $N_{b}=50, U=7 t$ which has only a $\mathrm{MI}$ in the middle.

To measure the total response of the system, summed over all momenta, we use

$$
S(\omega) \equiv \sum_{k} S(k, \omega)
$$

This quantity is shown in Fig. 6 for the system shown in Fig. 3(b) (same as Fig. 4) and also for $N_{b}=50, U=7 t$ which has pure MI in the center surrounded by SF at the edges. A two peak structure is seen where the first peak appears not to depend on $U$ whereas the second peak does. The first peak, therefore, is due to the SF component of the system and has an $\omega$ which corresponds very well to the saturation value of the SF branches of $S(k, \omega)$ in Figs. 3(a) and 3(b). On the other hand, the higher peaks depend on the value of $U$ and both correspond to the saturation values of the MI branches in $S(k, \omega)$ [see Fig. 3(b)]. Both peaks come from the high $k$ values of the SF and MI branches because those values have the larger spectral weights which therefore dominate when the sum over $k$ is taken. Recall that the $S(k, \omega)$ in the figures have been rescaled for better visibility and do not show the true spectral weights, but Fig. 6 does.

Our two-peak structure, Fig. 6, resembles the excitation spectrum in Fig. 2(a) in [3] for ${ }^{87} \mathrm{Rb}$ on one-dimensional lattices. Both quantities measure the total response of the system to a given frequency, but they are not the same and the connection between them is not clear. Although $S(k, \omega)$ does detect the presence of MI regions, it also shows there is no overall gap but that local excitation energies do correspond to gap energies in the uniform system at the same $U$. It is possible that the SF peak we detect is missed by Ref. [3] because the amplitude with which they shake their system is very large (20\% of the optical lattice potential).

We have presented the first Quantum Monte Carlo study of the dynamical structure factor, $S(k, \omega)$, for the Hubbard model on uniform and confined optical lattices. The uniform system exhibits linear dispersion (phonons) for the SF and a clear excitation gap in the MI in agreement with the directly computed gap [8]. In the absence of MI regions in the confined system, the $S(k, \omega)$ behavior is very similar to the uniform case.

However, when MI regions are present [Figs. 3(b) and 4], we find that although $S(k, \omega)$ shows the presence of such regions, it also clearly shows the absence of a gap: the system is globally compressible and gapless [11]. The presence of the MI regions is deduced from the presence of two excitation branches in $S(k, \omega)$ or from the double peak structure of $S(\omega)$ (Fig. 6). We note, as discussed above, that the second peak in Fig. 6 corresponds to MI regions. In addition, we note that $S(k, \omega)$ and $S(\omega)$ give information about local excitations in the trap. For example, by comparing $S(k, \omega)$ with $S(x, \omega)$ [Figs. 3(b) and 4] we have found that the first peak in $S(\omega)$ represents excitations in the SF regions at the edges of the system while the second peak represents the excitations in the MI regions. The value of the local excitation energy is consistent with the gap value for the uniform system at the same $U$. This is consistent with the local quantum criticality view $[11,16]$ for which we present new compelling evidence in Fig. 4. Our conclusions can be tested experimentally since the dynamic structure factor $S(k, \omega)$ (and therefore, $S(\omega)$ ) can be measured experimentally using Bragg spectroscopy [1,9].

We acknowledge helpful discussions with M. Troyer, H.T.C. Stoof, and C. Checker. R.T.S. was supported by NSFDMR-0312261 and NSF-INT-0124863, P.J.H.D. is supported by Stichting FOM.
[1] J. Stenger et al., Phys. Rev. Lett. 82, 4569 (1999); D. M. Stamper-Kurn et al., ibid. 83, 2876 (1999).

[2] M. Greiner et al., Nature (London) 415, 39 (2002).

[3] T. Stöferle et al., Phys. Rev. Lett. 92, 130403 (2004).

[4] B. Paredes et al., Nature (London) 429, 277 (2004).

[5] D. Jaksch et al., Phys. Rev. Lett. 82, 1975 (1999).

[6] D. Jaksch et al., Phys. Rev. Lett. 81, 3108 (1998).

[7] M. P. A. Fisher et al., Phys. Rev. B 40, 546 (1989).

[8] G. G. Batrouni et al., Phys. Rev. Lett. 65, 1765 (1990).

[9] J. Steinhauer et al., Phys. Rev. Lett. 88, 120407 (2002); D. van Oosten et al., Phys. Rev. A 71, 021601(R) (2005).
[10] R. Roth and K. Burnett, J. Phys. B 37, 3893 (2004).

[11] G. G. Batrouni et al., Phys. Rev. Lett. 89, 117203 (2002).

[12] K. S. D. Beach, cond-mat/0403055.

[13] J. K. Freericks and H. Monien, Phys. Rev. B 53, 2691 (1996).

[14] R. Roth and K. Burnett, Phys. Rev. A 67, 031602(R) (2003).

[15] We believe the irregularities on top of the overall structure of the spectral function to stem from the lack of translation invariance due to the trap. Hence, a particle-hole excitation of total momentum $k$ can decay into other particle-hole excitation of different total momenta which blurs the signal.

[16] M. Rigol et al., Phys. Rev. Lett. 91, 130403 (2003). 